\title{
Comunicação \\ Bacillus subtilis no tratamento de mudas de bananeira infectadas por fitonematoides ${ }^{1}$
}

\author{
Jardel Jean da Silva Araújo ${ }^{2}$, Maria de Fátima Silva Muniz ${ }^{2 *}$, Gilson Moura Filho², \\ Fernando da Silva Rocha ${ }^{3}$, José Mauro da Cunha e Castro ${ }^{4}$
}

10.1590/0034-737X201865010013

\section{RESUMO}

A utilização de material propagativo sadio constitui importante medida de controle para os nematoides da bananeira (Musa spp.), pois, assim, evita-se a introdução desses patógenos em áreas isentas. O objetivo deste trabalho foi avaliar o efeito da aplicação de Bacillus subtilis (Nemathel®) no tratamento de mudas, do tipo chifre, de bananeira subgrupo Terra cv. Comprida, infectadas por uma população mista com Radopholus similis, Meloidogyne spp., Pratylenchus spp. e Helicotylenchus spp. Foram testadas diferentes doses do produto (50; 100; 150; 200 e $250 \mathrm{~mL} / 10$ L de água) mais as testemunhas positiva (nematicida) e negativa (água). As mudas foram imersas em cada um dos tratamentos por 30 minutos e plantadas em vasos com solo esterilizado. A avaliação foi realizada após quatro meses e as doses de 200 e $250 \mathrm{~mL}$ do produto por $10 \mathrm{~L}$ de água proporcionaram as maiores reduções das populações dos nematoides em raízes e rizomas.

Palavras-chave: controle biológico; Musa spp.; rizobactérias; tratamento de mudas.

\section{ABSTRACT}

\section{Bacillus subtilis in the treatment of banana plantlets infected by nematodes}

The use of healthy propagative plant material is an important management method for nematodes in banana crops (Musa spp.), once it prevents the introduction of nematodes in non-infested areas. The aim of this work was to evaluate the effect of the bio-nematicide Nemathe ${ }^{\circledR}$ (Bacillus subtilis) on Terra banana plantlets cv. Comprida infected with a mixed population of Radopholus similis, Meloidogyne spp., Pratylenchus spp., and Helicotylenchus spp. Different doses of $\mathrm{Nemathe}^{\circledR}(50,100,150,200$, and $250 \mathrm{~mL} / 10 \mathrm{~L}$ water) were tested in addition to the positive (nematicide) and negative (water) treatments. The plantlets were immersed in each treatment for $30 \mathrm{~min}$ and subsequently, planted in pots containing sterilized soil in greenhouse. The evaluation was performed four months later. Dosages of 200 and 250 $\mathrm{mL}$ of $\mathrm{Nemathel}^{\circledR} / 10 \mathrm{~L}$ in water showed the greatest reduction of nematode populations in roots and rhizomes.

Key words: biological control; Musa spp.; rhizobacteria; treatment of plantlets.

Submetido em: 27/11/2015 e aprovado em 21/02/2018

2Universidade Federal de Alagoas, Centro de Ciências Agrárias, Rio Largo, Alagoas, Brasil. jean.jardel@gmail.com; mf.muniz@uol.com.br; gmf.ufal@yahoo.com.br

${ }^{3}$ Universidade Federal de Minas Gerais, Montes Claros, Minas Gerais, Brasil. rochafsplant@yahoo.com.br

${ }^{4}$ Embrapa Semiárido, Petrolina, Pernambuco, Brasil. mauro.castro@embrapa.br

*Autora para correspondência:mf.muniz@uol.com.br 


\section{INTRODUÇÃO}

Dentre os fatores bióticos que afetam a cultura da bananeira (Musa spp.), merecem destaque os nematoides Radopholus similis (Cobb) Thorne, Helicotylenchus multicinctus (Cobb) Golden, Pratylenchus coffeae (Zimmerman) Filipjev \& Schuurmans Stekhoven, Meloidogyne spp. e Rotylenchulus reniformis Linford \& Oliveira que infectam raízes e rizomas e causam perdas severas (Gowen et al., 2005).

As principais medidas de controle para esses nematoides incluem a utilização de mudas micropropagadas, rotação de culturas e aplicação de nematicidas químicos (Gowen et al., 2005). Por outro lado, bionematicidas à base de fungos e bactérias têm sido desenvolvidos e comercializados em todo o mundo, inclusive no Brasil (Ferraz et al., 2010).

Vários organismos são considerados antagonistas de fitonematoides, incluindo a rizobactéria Bacillus subtilis (Ehrenberg) Cohn. que podem agir de forma direta, afetando a eclosão ou a mobilidade dos juvenis. Seus antibióticos e toxinas são absorvidos pelos ovos dos nematoides e podem também agir de forma indireta, alterando os exsudatos das raízes ou induzindo a resistência sistêmica (Ferraz et al., 2010).

O efeito inibidor de isolados de B. subtilis em fitonematoides pode ser constatado por meio da microbiolização de sementes, com reduções na reprodução de Meloidogyne spp. nas culturas do tomateiro (Solanum lycopersicum L.) e arroz (Oryza sativa L.), (Ludwig et al., 2013; Fernandes et al., 2014). Essa bactéria apresenta, também, potencial em aplicação preventiva no tratamento de mudas micropropagadas de bananeira, para controle de M. javanica (Treub) Chitwood. Entretanto, não foram encontrados estudos envolvendo B. subtilis no tratamento de material propagativo naturalmente infectado (Lopes, 2011; Ribeiro et al., 2012).

O objetivo deste trabalho foi avaliar o efeito do tratamento de mudas convencionais de bananeira, infectadas por nematoides, com o bionematicida comercial à base de B. subtilis $\left(\mathrm{Nemathel}^{\circledR}\right)$.

\section{MATERIAL E MÉTODOS}

O experimento foi conduzido em casa de vegetação, no Centro de Ciências Agrárias da Universidade Federal de Alagoas - CECA/UFAL, de julho a novembro de 2014. Utilizaram-se mudas do tipo chifre, de bananeira subgrupo Terra cv. Comprida, provenientes de área infestada por fitonematoides.

A população inicial $(\mathrm{Pi})$ de nematoides foi estimada pela técnica de Coolen \& D’Herde (1972), em alíquotas de $10 \mathrm{~g}$ de rizoma/muda. Após a extração, os nematoides foram mortos e fixados em formaldeído, a 4\%, aquecido. A estimativa populacional foi realizada em lâmina de Peters, em microscópio de objetiva invertida, conforme as descrições de Mai \& Mullin (1996) e Mekete et al. (2012).

O delineamento experimental foi inteiramente casualizado, com sete tratamentos, constituídos por doses de Nemathel $^{\circledR}(50,100,150,200$ e $250 \mathrm{~mL} / 10$ L de água), além de água (testemunha negativa) e o nematicida Carbofurano (Furadan ${ }^{\circledR} 350$ SC) a $400 \mathrm{~mL}$ do produto comercial por 100 $\mathrm{L}$ de água (testemunha positiva). Foram utilizadas cinco repetições, cada uma constituída por uma muda, imersa, por período de 30 minutos, em calda com cada tratamento. Posteriormente, as mudas foram plantadas em vasos com $8 \mathrm{~L}$ de capacidade, que continham solo esterilizado em estufa $\left(100^{\circ} \mathrm{C} / 24 \mathrm{~h}\right)$.

Tabela 1: População inicial (Pi) de fitonematoides, em $10 \mathrm{~g}$ de rizoma; densidade populacional final (Pf), em $10 \mathrm{~g}$ de rizoma, em raízes e em $100 \mathrm{~cm}^{3}$ de solo e total; fator de reprodução (Pf/Pi), quatro meses após o plantio

\begin{tabular}{|c|c|c|c|c|c|c|}
\hline \multirow{2}{*}{ Tratamento } & \multirow{2}{*}{$\begin{array}{c}\text { População } \\
\text { Inicial }^{1}\end{array}$} & \multicolumn{3}{|c|}{ Densidade Populacional $^{1}$} & \multirow{2}{*}{$\begin{array}{c}\text { Densidade } \\
\text { Populacional Total }^{1} \\
\text { Raiz+Rizoma+Solo }\end{array}$} & \multirow{2}{*}{$\begin{array}{c}\text { Fator de Reprodução } \\
\text { Raiz+Rizoma+Solo }\end{array}$} \\
\hline & & Rizoma & Raiz & Solo & & \\
\hline Água & $1.005,3 \mathrm{a}$ & $85.840,0 \mathrm{a}$ & $91.013,3 \mathrm{a}$ & $21,3 \mathrm{a}$ & $176.874,6$ a & $207,7 \mathrm{a}$ \\
\hline $50 \mathrm{~mL}$ & 826,6 a & $8.000,0 \mathrm{~b}$ & $7.960,0 \mathrm{~b}$ & $38,6 \mathrm{a}$ & $15.332,0 \mathrm{~b}$ & $31,2 \mathrm{~b}$ \\
\hline $100 \mathrm{~mL}$ & $1.630,6 \mathrm{a}$ & $5.786,6 \mathrm{~b}$ & $6.786,6 \mathrm{~b}$ & $14,6 \mathrm{a}$ & $12.588,0 \mathrm{~b}$ & $36,7 \mathrm{~b}$ \\
\hline $150 \mathrm{~mL}$ & $1.382,7 \mathrm{a}$ & $5.680,0 \mathrm{~b}$ & $8.559,9 \mathrm{~b}$ & $21,3 \mathrm{a}$ & $14.261,3 \mathrm{~b}$ & $37,1 \mathrm{~b}$ \\
\hline $200 \mathrm{~mL}$ & $888,0 \mathrm{a}$ & $26,0 \mathrm{c}$ & $70,6 \mathrm{c}$ & $18,6 \mathrm{a}$ & $115,4 \mathrm{c}$ & $0,5 \mathrm{c}$ \\
\hline $250 \mathrm{~mL}$ & $1313,3 \mathrm{a}$ & $23,9 \mathrm{c}$ & $17,6 \mathrm{~d}$ & $33,3 \mathrm{a}$ & $74,9 \mathrm{c}$ & $0,1 \mathrm{c}$ \\
\hline Carbofurano & $1.316,0 \mathrm{a}$ & $1,1 \mathrm{~d}$ & $0,6 \mathrm{e}$ & $17,3 \mathrm{a}$ & $19,0 \mathrm{~d}$ & $0,03 \mathrm{c}$ \\
\hline$\overline{\mathrm{QMR}^{2}}$ & $0,0814^{\mathrm{ns}}$ & $0,0383 * *$ & $0,0298 * *$ & $0,1558^{\mathrm{ns}}$ & $0,0318 * *$ & $0,0580 * *$ \\
\hline $\mathrm{GLR}^{3}$ & 28 & 28 & 28 & 28 & 28 & 28 \\
\hline Média & 1194,62 & 15051,08 & 16344,10 & 23,59 & 31323,59 & 44,76 \\
\hline $\mathrm{CV}(\%)$ & 9,5 & 7,1 & 6,1 & 31,7 & 5,5 & 24,5 \\
\hline
\end{tabular}

${ }^{1}$ Dados transformados em $\log (x+1)$ para análise de variância. Valores seguidos da mesma letra, na coluna, não diferem entre si, pelo teste

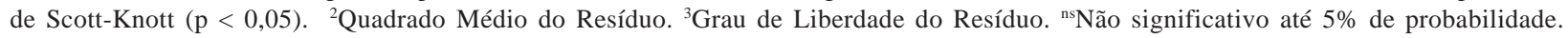
**Significativo até $1 \%$ de probabilidade. 
A avaliação do percentual de brotação das mudas e da população de nematoides no solo, rizomas e raízes ocorreu após quatro meses do plantio. Os nematoides foram extraídos de $100 \mathrm{~cm}^{3}$ de solo e de $10 \mathrm{~g}$ de cada tecido vegetal, utilizando-se as metodologias de Jenkins (1964) e de Coolen \& D’Herde (1972). O cálculo do fator de reprodução [FR = população final (raiz+rizoma+solo)/população inicial] foi determinado conforme Oostenbrink (1966).
Os dados foram transformados em $\log (\mathrm{x}+1)$ e submetidos à análise de variância. Para comparar os dados das testemunhas com as doses do bionematicida, foram utilizados o teste de Scott-Knott $(\mathrm{p}<0,05)$ e o software SAEG 5.0. Também foram realizadas análises de regressão da população dos nematoides, em solo, raízes e rizoma, da população final e do fator de reprodução, como variáveis dependentes das doses do bionematicida.
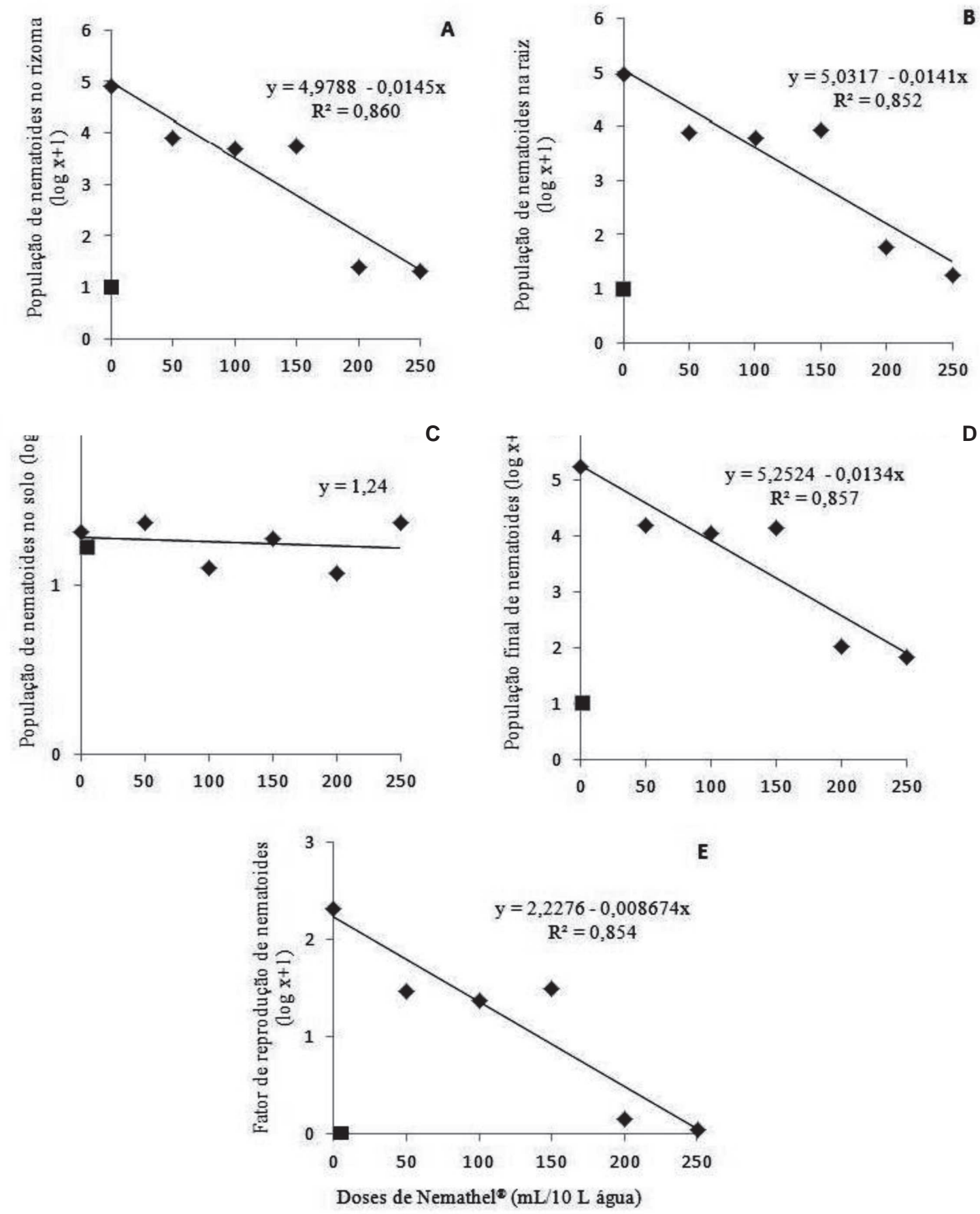

Figura 1: Populações de nematoides $(\log x+1)$, em resposta ao tratamento de mudas de bananeira com diferentes doses do bionematicida Nemathel ${ }^{\circledR}$ e do nematicida químico (ם). A) Rizoma, B) Raízes, C) Solo, D) População final, E) Fator de reprodução. 


\section{RESULTADOS E DISCUSSÃO}

A Pi de nematoides nos rizomas mostrou a presença de uma população mista, constituída por $R$. similis, Meloidogyne spp., Pratylenchus spp. e Helicotylenchus spp., com predomínio do último gênero e densidade populacional média dos tratamentos de 826,5 a 1630,62 espécimes/10 g de tecido. Não houve diferença estatística na avaliação da $\mathrm{Pi}$, demonstrando a uniformidade da infestação de nematoides nas repetições (Tabela 1). A percentagem de brotação das mudas foi de $100 \%$.

Em todos os tratamentos com o bionematicida, houve redução das populações finais de nematoides, quando comparadas com a testemunha negativa. Observaram-se as menores densidades populacionais nas doses de $200 \mathrm{e}$ $250 \mathrm{~mL}$ do produto por $10 \mathrm{~L}$ de água, com $\mathrm{FR}<1$, que se igualaram às do Carbofurano (Tabela 1). Verificou-se, também, significância na análise de regressão efetuada com o modelo linear para as variáveis avaliadas, exceto para a população dos nematoides no solo (Figura 1), confirmando-se que o aumento das doses do bionematicida afeta negativamente a população dos nematoides.

O potencial de $B$. subtilis para reduzir populações de nematoides ficou evidente, principalmente com espécies de Meloidogyne, em várias culturas, quando aplicado ao solo ou em microbiolização de sementes (Araújo \& Marchesi, 2009; Cardozo \& Araújo, 2011; Mutua et al., 2011; Ludwig et al., 2013; Fernandes et al., 2014).

Na cultura da bananeira, foi comprovada a redução das populações de Meloidogyne sp., H. multicinctus, $R$. similis e $P$. coffeae por micro-organismos antagonistas, incluindo B. subtilis aplicado ao solo em áreas de cultivo comercial (Guimarães, 2011). Lopes (2011) verificou redução na reprodução de $M$. javanica, em mudas micropropagadas, quando imersas em suspensões de células de rizobactérias, dentre as quais, B. subtilis, e, posteriormente, inoculadas com suspensão de ovos do nematoide. Ribeiro et al. (2012) também constataram a eficiência de $B$. subtilis em mudas micropropagadas e inoculadas com suspensão de juvenis de segundo estádio de $M$. javanica. Nesses trabalhos, os autores empregaram bananeira 'Prata-Anã', considerada resistente a $R$. similis, enquanto, neste trabalho, foi empregado o cultivar Comprida, susceptível a esse nematoide (Cordeiro et al., 2016).

A resposta de bananeiras à infecção por nematoides também depende do tipo de material propagativo empregado para o plantio. Mudas tradicionais comportaram-se como mais tolerantes, quando artificialmente inoculadas, com densidades populacionais de nematoides mais elevadas que aquelas obtidas por micropropagação (Blomme et al., 2004). Todavia, não se encontrou, na literatura científica, informação sobre a utilização de $B$. subtilis no tra- tamento de mudas convencionais naturalmente infectadas por nematoides, o que amplia as opções de manejo desses fitopatógenos na cultura da bananeira.

\section{CONCLUSÃO}

Bacillus subtilis aplicado por via do tratamento de mudas com Produto comercial Nemathel ${ }^{\circledR}$ nas doses de 200 e $250 \mathrm{~mL} / 10 \mathrm{~L}$ de água, reduziu a reprodução de fitonematoides em bananeira, com eficiência equiparada à do Carbofurano.

\section{AGRADECIMENTOS}

Os autores agradecem à Coordenação de Aperfeiçoamento de Pessoal de Nível Superior (Capes), pela concessão de bolsa ao primeiro autor, e à Empresa Caxiense de Controle Biológico Ltda (ECCB), pelo fornecimento do bionematicida.

\section{REFERÊNCIAS}

Araújo FF \& Marchesi GVP (2009) Uso de Bacillus subtilis no controle da meloidoginose e na promoção de crescimento do tomateiro. Ciência Rural, 39:1558-1561.

Blomme G, Beule H, Swennen RL, Tenkouano A \& Waele D (2004) Effect of nematodes on root and shoot growth of in vitro propagated and sword sucker-derived plants of six Musa spp. genotypes. Nematology, 6:593-604.

Cardozo RB \& Araújo FF (2011) Multiplicação de Bacillus subtilis em vinhaça e viabilidade no controle da meloidoginose, em cana-de-açúcar. Revista Brasileira de Engenharia Agrícola e Ambiental, 15:1283-1288.

Coolen WA \& D’Herde CJ (1972) A method for the quantitative extraction of nematodes from plant tissue. Ghent, State Agricultural Research Center. 77p.

Cordeiro ZJM, Matos AP \& Kimati H (2016) Doenças da bananeira. In: Amorim L, Rezende JAM, Bergamin Filho A \& Camargo LEA (Eds.) Manual de Fitopatologia: doenças das plantas cultivadas. $5^{\mathrm{a} e d .}$ Ouro Fino, Agronômica Ceres. p.109-123.

Fernandes RH, Vieira BS, Fuga CAG \& Lopes EA (2014) Pochonia clamydosporia e Bacillus subtilis no controle de Meloidogyne incognita e $M$. javanica em mudas de tomateiro. Bioscience Journal, 30:194-200.

Ferraz S, Freitas LG, Lopes EA \& Dias-Arieira CR (2010) Manejo sustentável de fitonematoides. Viçosa, Editora UFV. 306p.

Gowen SR, Quénéhervé P \& Fogain R (2005) Nematodes of banana and plantains. In: Luc M, Sikora R \& Bridge J (Eds.) Plant parasitic nematodes in subtropical and tropical agriculture. $2^{\mathrm{a}}$ ed. London, CAB International. p.611-643.

Guimarães CP (2011) Controle biológico de fitonematoides na cultura da banana no norte de Minas Gerais. Dissertação de Mestrado. Universidade Estadual de Montes Claros, Montes Claros. 90p.

Jenkins WR (1964) A rapid centrifugal-flotation technique for separating nematodes from soil. Plant Disease Reporter, 48:692.

Lopes OS (2011) Aplicação de rizobactérias em explantes e plântulas de bananeira 'prata-anã' no controle de Meloidogyne javanica e no desenvolvimento de mudas. Dissertação de Mestrado. Universidade Estadual de Montes Claros, Montes Claros. 123p. 
Ludwig J, Moura AB \& Gomes CB (2013) Potencial da microbiolização de sementes de arroz com rizobactérias para o biocontrole do nematoide das galhas. Tropical Plant Pathology, 38:264-268.

Mai WF \& Mullin PG (1996) Plant-parasitic nematodes: a pictorial key to genera. $5^{\text {th }}$ ed. New York, Cornell University. 277p.

Mekete T, Dababat A, Sekora N, Akyazi F \& Abebe E (2012) Identification key for agriculturally important plant-parasitic nematodes: a manual for nematology. Mexico, CIMMYT. 22p.

Mutua GK, Karanja NK, Ayuke F, Ndukhu H \& Kimenju JW (2011) The potential of Bacillus subtilis and Rhizobium leguminosarum in controlling plant-parasitic nematodes in farmers' fields. African Crop Science Conference Proceedings, 10:209-215.
Oostenbrink M (1966) Major characteristics of the relation between nematodes and plants. Wageningen, Mededelingen Landbouwhogeschool. 46p.

Ribeiro RCF, Campos VP, Xavier AA, Rocha LS, Ribeiro HB, Aguiar FM, Souza RM, Mizobutsi EH \& Dias-Arieira CR (2012) Rizobactérias no controle de Meloidogyne javanica e mal do Panamá em bananeira. Nematropica, 42:218-226. 